\title{
Improvement in Sensitivity of Live Cell-Based Sensor Cells by Co- Transfection of a Reporter Gene Driven by a Modified HSP70B' and HSF1 Expression Vector
}

\author{
Ken-Ichi Wada, Junko Okuda-Shimazaki and Akiyoshi Taniguchi ${ }^{*}$ \\ Cell-Sensing Group, Biomaterials Center, National Institute for Materials Sciences, 1-1, Namiki, Tsukuba, Ibaraki $305-$ \\ 0044, Japan
}

\begin{abstract}
We previously reported that live cells transfected with a luciferase construct carrying the HSP70B' gene promoter increases the luciferase activity in response to toxic reagents and can be used as an intelligent cytotoxicity sensor device (i.e., sensor cells). In the present study, in order to improve the sensitivity of these cytotoxic sensor cells, we cotransfected the sensor cells with both the luciferase construct carrying the HSP70B' gene promoter (pMCREx3) and an HSF1 expression vector driven by a modified HSP70B' promoter (pCRE/HSF). Cells co-transfected with pMCREx3 and pCRE/HSF showed 2.5 times higher sensitivity for $\mathrm{CdCl}_{2}$ cytotoxicity than cells transfected with pMCREx3 alone, indicating that sensor cells co-transfected in this way are useful and sensitive tools with which to detect a cytotoxic response.
\end{abstract}

Keywords: HSF, HSP70B' promoter, luciferase assay, sensor cell.

\section{INTRODUCTION}

Biosensors, or sensors utilizing biological components, have attracted a great deal of attention because of their highspecificity and sensitivity for their targets $[1,2]$. In particular, live cell-based biosensors can potentially report functional information about the effects of cytotoxic stimulation and are applicable in many fields such as toxicology, environmental assessment, and drug screening. One method to obtain functional information from live cells is to monitor gene expression levels using reporter assays with certain promoters.

The heat shock protein 70B' (HSP70B') gene was cloned by Lueng et al. as a novel stress-inducible HSP gene [3]. The mRNA expression of inducible HSP genes including HSP70B' is strongly induced by various cellular stresses, such as mechanical stress [4], alkaline shift [5], copper and zinc [6], heavy metals [7], antibiotics [8], anticancer drugs [9], and other toxic reagents [10-12]. Additionally, the basal expression level of the HSP70B' gene is lower than that of other HSP70 genes [13]. These facts suggest that the HSP70B' gene promoter has advantages over other HSP genes for construction of cytotoxicity sensor cells.

We previously reported that live cells transfected with a luciferase construct carrying the HSP70B' gene promoter can be used as a biosensor for cytotoxicity (i.e., sensor cells), and these sensor cells increase their luciferase activity in response to a cytotoxic reagent [14]. Naturally, improvement in the cytotoxic response of these sensor cells would lead to more reliable live cell-based cytotoxicity sensors.

The up-regulation of HSP genes in response to toxic reagents is mediated by heat shock factor (HSF). Cellular stresses induce HSF activation, resulting in the formation of the HSF triplet, which binds to the heat shock element (HSE)

*Address correspondence to this author at the Cell-Sensing Group, Biomaterials Center, National Institute for Materials Sciences, 1-1, Namiki, Tsukuba, Ibaraki 305-0044, Japan; Tel: +81-29-860-4505; Fax: +81-29-8604714; E-mail: TANIGUCHI.Akiyoshi@nims.go.jp located in the HSP genes promoter and increases HSP gene transcriptional activity [15-17]. Thus, multiplication of HSF and/or HSE has the potential to improve the cytotoxic response of HSP gene promoter-based sensor cells. We have already found the cytotoxic responding element (CRE) in HSP70B' gene promoter, in which functional HSEs are contained and AP-1 site is removed by base substitution from AGT to CAG. The transfection of luciferase constructs driven by modified HSP70B' promoter, which contains tandem repeats of CRE in front of the HSP70B' promoter resulted in fabrication of highly sensitive cytotoxicity sensor cells [18]. In addition it is expected that an increase in the HSF will further improve the cytotoxic response in the sensor cells.

The aim of the present study, which we achieved, was improvement of the cytotoxic response in HSP70B' promoter-based sensor cells by co-transfection of HSF1 expression vector.

\section{MATERIALS AND METHODS}

\section{Cell Culture}

A human liver carcinoma cell line (HepG2) was cultured in Dulbecco's modified Eagle's medium containing 4,500 $\mathrm{mg} / \mathrm{mL}$ glucose and supplemented with $10 \%$ fetal bovine serum, $100 \mathrm{U} / \mathrm{mL}$ penicillin and $100 \mathrm{mg} / \mathrm{mL}$ streptomycin. Cultured cells were maintained at $37^{\circ} \mathrm{C}$ in a humidified atmosphere containing $5 \% \mathrm{CO}_{2}$.

\section{Construction of Plasmids}

The structures of the plasmids used in the present study are shown in Fig. (1). pMCREx3 was prepared in our previous study [18]. pCRE/HSF was prepared as follows; the open reading frame of the human HSF1 gene was amplified by standard PCR method from human umbilical vein endothelial cell (HUVEC) cDNA using 5'-ATGGATCTGCCCGT GGGCCC-3' and 5'-CTAGGAGACAGTGGGGTCCTTGG CTTT-GGGAAA-3' as the forward and reverse primers, respectively. The HSF1 gene fragment was replaced with the 
CMV promoter/EGFP gene region in pd2EGFP (Clontech, CA). Next, the modified HSP70B' gene promoter fragment was isolated from pMCREx3 and inserted in front of the HSF1 gene.

\section{Transfection and Luciferase Assay}

Transfection into HepG2 cells then followed. The cells were seeded in 24-well plates at a density of approximately $8 \times 10^{4}$ cells per well. One day after cell seeding, a mixture of pMCREx3 and pCRE/HSF or pMCREx3 alone was transfected into the cells using SuperFect Reagent (Qiagen GmbH., Hilden, Germany) for 12 hours. pRL-CMV plasmid, which contains the CMV promoter upstream from the Renilla luciferase gene (Promega, WI), was also transfected to serve as an internal control for variations in transfection efficiency. The sensor cells were then harvested at $0,6,12$, 24 , and $48 \mathrm{~h}$ after the addition of $\mathrm{CdCl}_{2}$. The luciferase assay was performed using the Dual-Luciferase Reporter Assay System (Promega) as previously described [18,19].

The luciferase activities were represented as the relative values compared to the luciferase construct driven by SV40 promoter $(\% \mathrm{SV} 40)$, and the cytotoxic response was defined as folds induction compared between cells treated with and without $\mathrm{CdCl}_{2}$ treatment. The increase ratio of the luciferase activity in the sensor cells was calculated from the difference in the luciferae value measured in 100 and $500 \mathrm{ng} / \mathrm{mL} \mathrm{CdCl}_{2}$ exposure for $12 \mathrm{~h}$. The detection limit of the sensor cells for $\mathrm{CdCl}_{2}$ cytotoxicity was determined as the minimal $\mathrm{CdCl}_{2}$ concentration which induces increase in luciferase activity in the sensor cells above the detection threshold. The detection threshold was defined as the average luciferase value plus two times of the standard deviation measured in the sensor cells treated with normal growth medium.

\section{RESULTS}

\section{Increase in Cytotoxic Response Upon pCRE/HSF Co- Transfection}

The cells transfected with the luciferase construct driven by SV40 promoter showed no increase in luciferase activity in response to $\mathrm{CdCl}_{2}$ exposure (data not shown). We previously constructed sensor cells using pMCREx3 plasmid [18]. The pMCREx 3 plasmid encodes the luciferase gene under the control of a modified HSP70B' promoter, in which the cytotoxicity responding element (CRE) is repeated three times in front of the HSP70B' gene promoter (Fig. 1). In order to produce more sensitive sensor cells, the luciferase reporter plasmid driven by modified HSP70B' promoter and an HSF1 expression vector driven by modified HSP70B' promoters (pMCREx3 and pCRE/HSF, respectively; Fig. 1) were co-transfected into HepG2 cells. The cytotoxic response of the co-transfected cells was measured $12 \mathrm{~h}$ after exposure $100 \mathrm{ng} / \mathrm{mL} \mathrm{CdCl}_{2}$ (Fig. 2). We used $\mathrm{CdCl}_{2}$ as the cytotoxic stimulus because it is widely used as a model toxic heavy metal compound and is a powerful inducer of the HSP gene [20]. Cells transfected with pMCREx3 alone showed only a 1.3 -fold increase in response to $\mathrm{CdCl}_{2}$ exposure. In contrast, cells co-transfected with pMCREx3 and 1, 10, and $100 \mathrm{ng} /$ well pCRE/HSF showed a 2.5-, 2.6-, and 2.3-fold increase in cytotoxcic response, respectively. There were no significant differences in cytotoxic response among these cotransfected cells. The results indicate that the cytotoxic response of pMCREx3-containing sensor cells was increased by and pCRE/HSF co-transfection.

\section{Time Course Change in Luciferase Activity of Co- Transfected Cells}

In order to analyze the effect of pCRE/HSF cotransfection on the time course of the change in luciferase activity, we measured the luciferase activity at $0,6,12,24$, and $48 \mathrm{~h}$ after $\mathrm{CdCl}_{2}$ exposure in HepG2 cells transfected with pMCREx3 alone or co-transfected with pMCREx3 plus pCRE/HSF (Fig. 3). Both types of transfected cells showed increased luciferase activity that peaked at $12 \mathrm{~h}$ after initial $\mathrm{CdCl}_{2}$ exposure and then decreased. However, a smaller decrease in luciferase activity was observed in the cotransfected cells. At $48 \mathrm{~h}$ after $\mathrm{CdCl}_{2}$ exposure, cotransfected cells showed $48 \%$ of peak luciferase activity, while cells transfected with pMCREx3 alone showed only 11 $\%$ of peak luciferase activity. Both co-transfected and cells transfected with pMCREx3 alone showed a smaller increase in luciferase activity at all time points during the first $48 \mathrm{~h}$ when treated with normal growth medium.

\section{Sensitivity of Sensor Cells for Cytotoxicity}

The standard curve of $\mathrm{CdCl}_{2}$ concentration using cells cotransfected with pMCREx 3 and pCRE/HSF indicates that the luciferase activity of the co-transfected cells increased in a

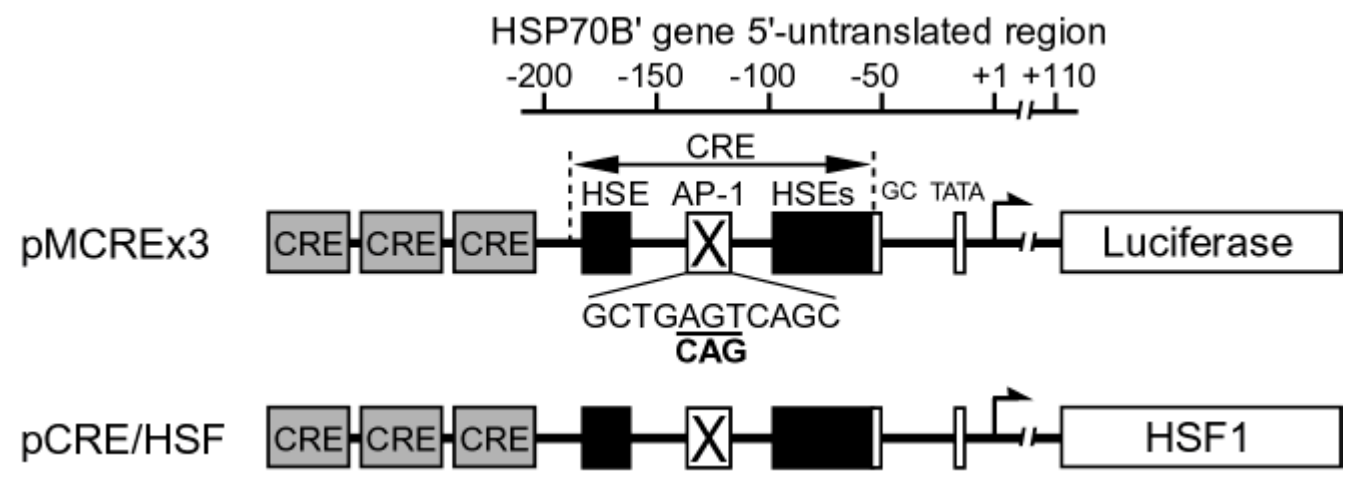

Fig. (1). The structure of the pMCREx 3 and pCRE/HSF plasmids. The cytotoxic responding element (CRE) is located in nts-192 to -56 in the HSP70B' gene 5'-untranslated region. In the CRE, the AGT nucleotides located in the center of the AP-1 biding site were changed to CAG. The modified HSP70B' gene promoter has three tandem repeats of CRE in front of thr HSP70B' gene promoter. Both the luciferase and HSF1 genes are under the control of the modified HSP70B' gene promoters (pMCREx3 and pCRE/HSF, respectively). 

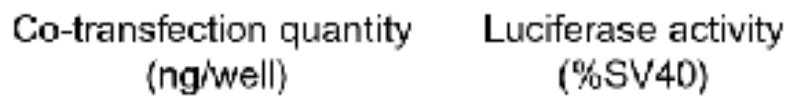

\section{Cytotoxic response \\ (Fold-increase)}
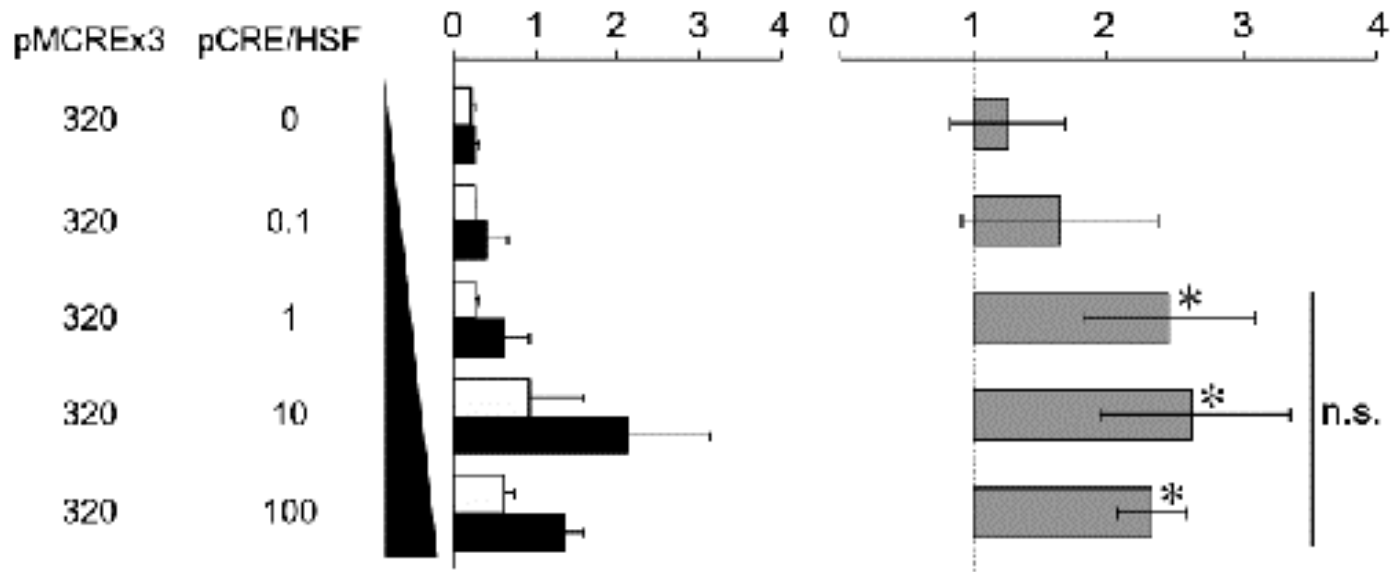

Fig. (2). Effect of co-transfection of pCRE/HSF on cytotoxic response. The co-transfection quantities are shown at left. Center panel shows the luciferase activity in the transfected cells treated with normal growth medium (white bars) or with growth medium containing $100 \mathrm{ng} / \mathrm{mL}$ $\mathrm{CdCl}_{2}$ (black bars) after $12 \mathrm{~h}$ initial exposure. The luciferase activities are indicated as values relative to the SV40 promoter. Right panel shows cytotoxic response in the transfected cells represented as fold of induction (gray bars). Values represent mean \pm SD. ${ }^{*}$ Significantly different from the cells without pCRE/HSF co-transfection. n.s.: not significant. $(n=3, p<0.05)$.

dose-dependant manner up to at least $5.46 \mathrm{nmol} / \mathrm{mL} \mathrm{CdCl}_{2}$ $(1,000 \mathrm{ng} / \mathrm{mL})$, with a detection limit for $\mathrm{CdCl}_{2}$ of 380 $\mathrm{pmol} / \mathrm{mL}$ (70 ng/mL) (Fig. 4). The increase ratio of the standard curve using cells co-transfected with pMCREx3 and pCRE/HSF was $0.2 \% \mathrm{SV} 40 / \mathrm{CdCl}_{2} \mathrm{ng}$, while the increase ratio of the standard curve using cells transfected with pMCREx3 alone was shown in a previous study to be $0.08 \%$ $\mathrm{SV} 40 / \mathrm{CdCl}_{2}$ ng [18]. These data indicate that cells cotransfected with pMCREx3 and pCRE/HSF have 2.5 timeshigher sensitivity for $\mathrm{CdCl}_{2}$ cytotoxicity than cells trans- fected with pMCREx3 alone. However, the detection limit for $\mathrm{CdCl}_{2}$ using cells co-transfected with pMCREx3 and pCRE/HSF was the same as with pMCREx3 alone. In total these results indicate that cells co-transfected with pMCREx3 and pCRE/HSF can perform highly sensitive and quantitative cytotoxicity detection.

\section{DISCUSSION}

We report herein the successful improvement of the cytotoxic sensitivity of sensor cells by co-transfection of a lu-

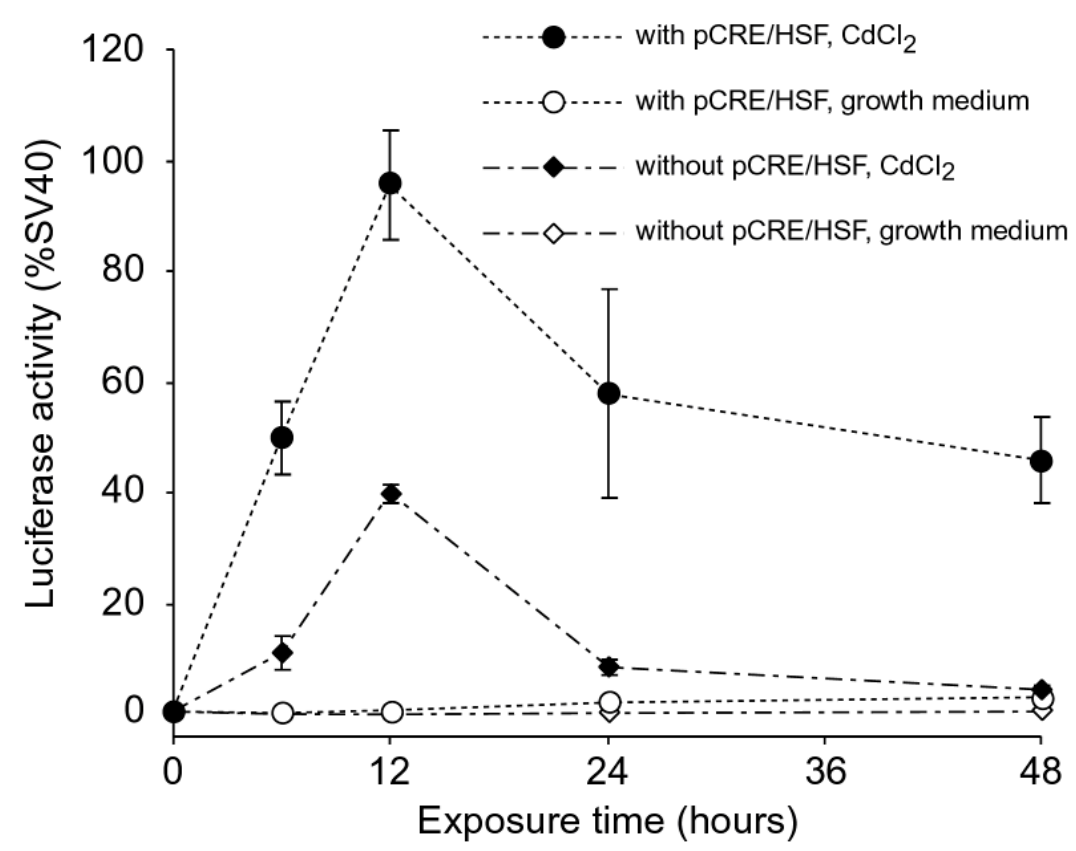

Fig. (3). Time course change in luciferase activity in sensor cells. Circles and diamonds represent the luciferase activity in the HepG2 cells co-transfected with pCRE/HSF and pMCREx3 (100 ng pCRE/HSF and $320 \mathrm{ng}$ pMCREx3/well) and transfected with pMCREx3 alone (320 ng pMCREx3/well), respectively. The transfected cells were treated with growth medium containing $500 \mathrm{ng} / \mathrm{mL} \mathrm{CdCl}_{2}$ (filled symbols) or normal growth medium (open symbols). The luciferase activities are indicated as values relative to the SV40 promoter. Values represent mean \pm SD. $(n=3)$. 


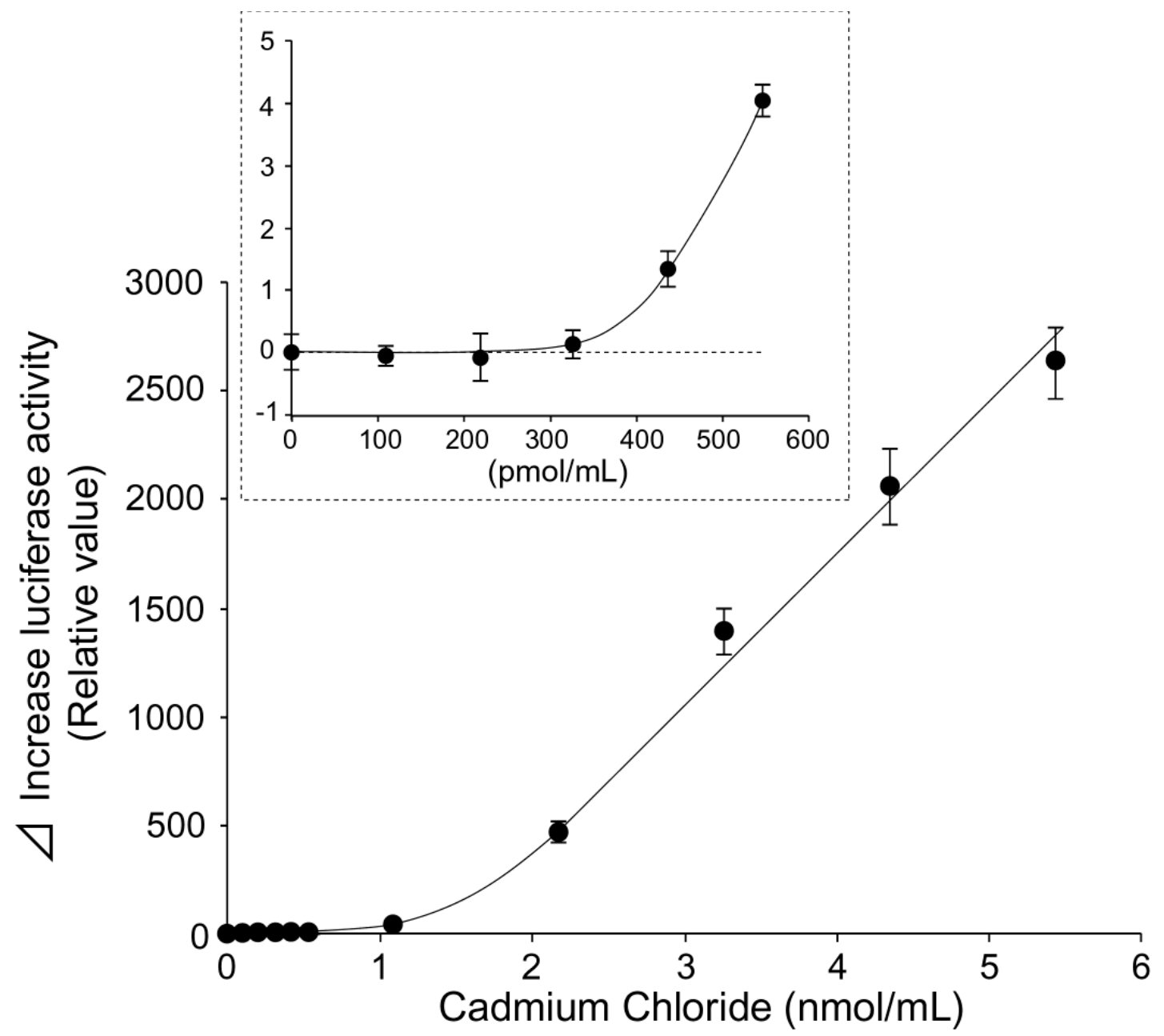

Fig. (4). Standard curve for detection of $\mathrm{CdCl}_{2}$ cytotoxicity. The luciferase activities were measured in the co-transfected cells using 100 ng pCRE/HSF and $320 \mathrm{ng}$ pMCREx3/well after $12 \mathrm{~h}$ of $\mathrm{CdCl}_{2}$ exposure. The luciferase activities in low concentrations of CdCl $\mathrm{Cxposure}_{2}$ are magnified in the box. The relative luciferase activities are indicated as the difference from the value in growth medium treatment (delta increase). Values are represented mean \pm SD. $(n=4)$.

ciferase construct carrying the HSP70B' gene promoter with an HSF1 expression vector driven by a modified HSP70B' promoter. In mammals, the HSF family encompasses at least three genes including HSF1, HSF2, and HSF4 [16]. Although each HSF forms a triplet and binds to HSE, it is generally thought that the stress response in live cells associated with the HSP genes is mainly regulated by HSF1. Thus, in the present study, we tried to improve the cytotoxic response of the HSP70B' promoter-based sensor cells by cotransfection with our usual construct, pMCREx3, together with an HSF1 expression vector driven by a modified HSP70B' promoter, pCRE/HSF. We found 2.5 times-higher sensitivity for $\mathrm{CdCl}_{2}$ cytotoxicity with co-transfection than with transfection with pMCREx3 alone.

The maximum luciferase activity in the co-transfected cells was observed at $12 \mathrm{~h}$ after initial $\mathrm{CdCl}_{2}$ exposure, the same as for cells transfected with pMCREx3 alone, indicating no improvement in the detection time by co-transfection. In HSP70B' promoter-based sensor cells, a lag time in the detection of cytotoxicity after exposure to the toxic reagent is inevitable, as several essential steps are required to increase luciferase activity including transcription, translation, maturation, and accumulation of luciferase. On the other hand, real time monitoring of the HSF conformational change by fluorescence resonance energy transfer (FRET) technology in live cells $[21,22]$ using fused HSF and fluorescent proteins will provide a system to produce live cell-based cytotoxicity sensors that can report the cytotoxicity immediately after exposure to the toxic reagent.

Cells have detoxification system against heavy metals such as scavenge by metallothioneins or glutathione, and active export by P-type ATPases or MRP2 [23]. The efficiency of these detoxification system is different among cell types and heavy metals. Indeed, we have observed that Arsenic cytotoxicity is lower than Cadmium, and that the HSP70B' promoter-based sensor cells fabricated in NIH-3T3 cells show lower cytotoxic response to $\mathrm{CdCl}_{2}$ than that of fabricated in HepG2 cells (data not shown).

The HSP promoter-based sensor cells have a potential to detect the general cytotoxic stimulations accompany with protein denature. However, HSP genes promoter does not respond against some other toxicants such like mutagens which induce DNA damage. In this case, p53 target gene promoter-based sensor cells will detect the cytotoxicity of mutagens. 
In summary, in the present study, we co-transfected a luciferase construct carrying the HSP70B' gene promoter with an HSF1 expression vector driven by a modified HSP70B' promoter to produce improved cytotoxicity sensor cells. The improved sensor cells 1) showed increased luciferase activity in response to $\mathrm{CdCl}_{2}$ in a dose-dependent manner, 2) maintained the increased luciferase activity for at least $48 \mathrm{~h}$ after initial $\mathrm{CdCl}_{2}$ exposure, and 3) showed high sensitivity for $\mathrm{CdCl}_{2}$. These sensor cells can be used as a reliable cytotoxic biosensor for use in toxicology, environmental assessment, and drug screening.

\section{REFERENCES}

[1] Bousse L. Whole cell biosensors. Sensor Actuat B-Chem 1996; B 34: $270-5$.

[2] Keusgen M. Biosensors: new approaches in drug discovery. Naturwissenschaften 2002; 89: 433-44.

[3] Leung TK, Rajendran MY, Monfries C, Hall C, Lim L. The human heat-shock protein family. Expression of a novel heat-inducible HSP70 (HSP70B') and isolation of its cDNA and genomic DNA. Biochem J 1990; 267: 125-32.

[4] Xu Q, Schett G, Li C, Hu Y, Wick G. Mechanical stress-induced heat shock protein 70 expression in vascular smooth muscle cells is regulated by Rac and Ras small $\mathrm{G}$ proteins but not mitogenactivated protein kinases. Circ Res 2000; 86: 1122-8.

[5] Petronini PG, Alfieri R, Campanini C, Borghetti AF. Effect of an alkaline shift on induction of the heat shock response in human fibroblasts. J Cell Physiol 1995; 162: 322-9.

[6] Hatayama T, Asai Y, Wakatsuki T, Kitamura T, Imahara H. Regulation of hsp70 synthesis induced by cupric sulfate and zinc sulfate in thermotolerant HeLa cells. J Biochem (Tokyo) 1993; 114: 592-7.

[7] Levinson W, Oppermann H, Jackson J. Transition series metals and sulfhydryl reagents induce the synthesis of four proteins in eukaryotic cells. Biochim Biophys Acta 1980; 606: 170-80.

[8] Romano CC, Benedetto N, Catania MR, Rizzo A, Galle F, Losi E, Hasty DL, Rossano F. Commonly used antibiotics induce expression of Hsp 27 and Hsp 60 and protect human lymphocytes from apoptosis. Int Immunopharmacol 2004; 4: 1067-73.

[9] Ait-Aissa S, Porcher J, Kretz-Remy C, Velarde G, Arrigo A, Lambre C. Induction of the hsp70 gene promoter by various anticancer drugs. Toxicol In Vitro 1999; 13: 651-55.

[10] Tchounwou PB, Ishaque AB, Schneider J. Cytotoxicity and transcriptional activation of stress genes in human liver carcinoma cells
(HepG2) exposed to cadmium chloride. Mol Cell Biochem 2001; 222: 21-8.

[11] Byrne CR, Hanson PJ. Induction of heat shock protein 72 by a nitric oxide donor in guinea-pig gastric mucosal cells. Eur J Pharmacol 1998; 353: 117-22.

[12] Ito H, Hasegawa K, Inaguma $\mathrm{Y}$, Kozawa O, Asano T, Kato K. Modulation of the stress-induced synthesis of stress proteins by a phorbol ester and okadaic acid. J Biochem (Tokyo) 1995; 118: 62934.

[13] Parsian AJ, Sheren JE, Tao TY, Goswami PC, Malyapa R, Van Rheeden R, Watson MS, Hunt CR. The human Hsp70B gene at the HSPA7 locus of chromosome 1 is transcribed but non-functional. Biochim Biophys Acta 2000; 1494: 201-5.

[14] Wada K, Taniguchi A, Xu L, Okano T. Rapid and highly sensitive detection of cadmium chloride induced cytotoxicity using the HSP70B' promoter in live cells. Biotechnol Bioeng 2005; 92: 4105.

[15] Wu C. Heat shock transcription factors: structure and regulation. Annu Rev Cell Dev Biol 1995; 11: 441-69.

[16] Morimoto RI, Wu C. Regulation of the heat shock transcriptional response: cross talk between a family of heat shock factors, molecular chaperones, and negative regulators. Genes Dev 1998; 12: 3788-96.

[17] Kiang JG, Tsokos GC. Heat shock protein $70 \mathrm{kDa}$ : molecular biology, biochemistry, and physiology. Pharmacol Ther 1998; 80: 183201.

[18] Wada KI, Taniguchi A, Okano T. Highly sensitive detection of cytotoxicity using a modified HSP70B' promoter. Biotechnol Bioeng 2007; 97: 871-876.

[19] Xu L, Harada H, Yokohama-Tamaki T, Matsumoto S, Tanaka J, Taniguchi A. Reuptake of extracellular amelogenin by dental epithelial cells results in increased levels of amelogenin mRNA through enhanced mRNA stabilization. J Biol Chem 2006; 281: 2257-62.

[20] Wu BJ, Kingston RE, Morimoto RI. Human HSP70 promoter contains at least two distinct regulatory domains. Proc Natl Acad Sci U S A 1986; 83: 629-33.

[21] Heyduk T. Measuring protein conformational changes by FRET/LRET. Curr Opin Biotechnol 2002; 13: 292-6.

[22] Zaccolo M. Use of chimeric fluorescent proteins and fluorescence resonance energy transfer to monitor cellular responses. Circ Res 2004; 94: 866-73.

[23] Ballatori N. Transport of toxic methods by molecular minicry. Environ Health Perspect 2002; 110: 689-94. 